\title{
COMPUTER TECHNOLOGY
}

\section{An X Windows tool for synthesizing face images from eigenvectors}

\author{
ALICE J. O'TOOLE and JAMIE L. THOMPSON \\ University of Texas at Dallas, Richardson, Texas
}

\begin{abstract}
An X Windows software tool for the construction of faces with a weighted combination of eigenvectors is described. The eigenvectors were extracted from an autoassociative matrix that com. prised 100 face images. The program input consists of eigenvectors and sets of weights that describe individual faces and combines these to create face images. The tool creates a panel of buttons that permits the display of individual eigenvectors and the display of an average face as well. Facilities for on-line changes to the intensity of individual eigenvectors can be used to change the appearance of a face. Previously, O'Toole, Abdi, Deffenbacher, and Bartlett (1991) have shown that the intensity of certain individual eigenvectors contains reliable information for determining the sex and race of the face.
\end{abstract}

The complicated visual pattern of the human face is difficult to quantify in traditional feature-based or verbal descriptive terms. Faces are highly similar to one another in that they all contain the same set of "features" (i.e., eyes, nose, and mouth) arranged in roughly the same configuration. Thus, in order to distinguish familiar from unfamiliar faces, people must be able to make use of very subtle variations in the form and configuration of facial features. Yet, despite these computational problems, a good deal of empirical evidence indicates that human face recognition skills are impressive (e.g. , Bahrick, Bahrick, \& Wittlinger, 1975). For the problem of recalling a face from memory, however, human capabilities are somewhat more difficult to assess. The long-standing problem that police have in creating an image of a crime suspect's face from a witness' verbal description, and the problems that witnesses have in creating an Identikit/Photofit composite both testify to this fact. Both composite and verbal descriptions are limited as means of creating a natural likeness of a face. For example, empirical studies (see Davies, 1982, for an excellent review), indicate that composite techniques generally do not produce good enough likenesses of a face for subjects to accurately match a composite to the face from which it was made.

In this paper, we describe software for synthesizing face images via a more global approach to quantifying the information in faces. Although our program is not meant as a solution for the basic problem of face recall, it does provide a procedure for creating and altering a face by using "features" that allow for the synthesis of more

Thanks are due June Chance and $\mathrm{Al}$ Goldstein, for providing the faces used in the simulations, and Dominique Valentin, Candice Walker, and three anonymous reviewers, for comments on an earlier version of this manuscript. Correspondence should be addressed to A.J. O'Toole, School of Human Development, University of Texas at Dallas, Richardson, TX 75083-0688 (e-mail otoole@utdallas.edu). natural-looking faces than those that can be created via composite techniques. Our approach builds on an observation made by Kohonen (1977), who demonstrated that an autoassociative network that comprised pixel codings of face images could act as a content-addressable memory for faces. In addition, he showed that faces stored in this memory could be reconstructed through the use of incomplete or noisy memory retrieval keys. This approach has been used in a number of recent attempts to model face recognition and categorization. For example, O'Toole, Millward, and Anderson (1988) have shown that the model can distinguish learned from novel faces with a high degree of accuracy. Furthermore, with differential amounts of training on two races of faces, the model is able to simulate the difficulties humans have in recognizing other-race faces ( $O$ 'Toole, Deffenbacher, Abdi, \& Bartlett, 1991). Variations of the basic principles of this model have been applied to the problem of categorizing faces by sex (Golomb, Lawrence, \& Sejnowski, 1991; Flemming \& Cottrell, 1990) and by race and sex (O'Toole, Abdi, Deffenbacher, \& Bartlett, 1991).

Yet, although this approach to quantifying the information in faces may offer insight into a number of interesting psychological questions, the manipulation of this information (i.e., eigenvectors) is awkward and difficult. The software described here was created to make such information easy to manipulate and more widely available to researchers interested in how an eigenvector description of a face pattern is different from the more readily available composite face construction systems. This paper is organized as follows. First, we define the autoassociative matrix memory and then show how faces can be described as a weighted sum of the eigenvectors extracted from this matrix. Second, we give specifics of simulations that provide the input for the software tool. Third, we give details of the program's capabilities for synthesizing and experimenting with the eigenvectors and faces. 
Fourth, we discuss some potential applications of this tool in the study of face processing. We conclude with a short description of the specifics of the program and its portability.

\section{Model Definition}

An autoassociative memory matrix is constructed as the sum of outer product matrices for a set of stimuli coded as vectors. For images such as faces, an autoassociative memory matrix can be constructed as follows:

$$
\mathbf{A}=\sum_{i} \mathbf{f}_{i} \mathbf{f}_{i}^{T},
$$

where $\mathbf{f}_{i}$ is the $i$ th face, coded as a pixel vector consisting of the concatenation of the rows of the face image, and where the faces are assumed to be normalized vectors (i.e., $\mathbf{f}_{i}^{T} \mathbf{f}_{i}=1$ ). Thus, $A$ can be thought of as a pixel-bypixel matrix that represents a kind of composite memory of the faces. A simple neural network interpretation of this linear autoassociator is that each element $\mathbf{A}_{i j}$ of the matrix contains the connection strength between the $i$ th and $j$ th neurons. This is a distributed rather than a localized code, since the representation of any given face is not found in a localized part of the memory but is distributed across the connection strengths throughout the entire memory.

The $i$ th face can be recalled from this memory matrix as follows:

$$
\hat{\mathbf{f}}_{i}=\mathbf{A} \mathbf{f}_{i},
$$

where $\hat{\mathbf{f}}_{i}$ is the system estimate of $\mathbf{f}_{i}$. A neural network interpretation of this process can be seen as follows. Each element $j$ of the retrieved $i$ th face vector $\hat{\mathbf{f}}_{i}$ is interpretable as the output of the $j$ th neuron. Since this neuron is connected to all other neurons, its output is simply the sum of its inputs (the face vector), weighted by the connection strengths between itself and all of the neurons to which it connects. These connection strengths are established by the statistical properties of the input set of faces via the pixel co-occurrence structure captured by the summed outerproducts in $\mathbf{A}$.

The quality of the output face estimate is measured by comparing the reconstructed image with the original image, using the cosine of the angle between the vectors $\hat{\mathbf{f}}_{i}$ and $\mathbf{f}_{i}$. Like any square, symmetrical matrix, the matrix A can be expressed as a weighted sum of the outerproducts of its eigenvectors (see, e.g., Jackson, 1991):

$$
\mathbf{A}=\sum_{i} \lambda_{i} \mathbf{e}_{i} \mathbf{e}_{i}^{T},
$$

where $\lambda_{i}$ is the $i$ th eigenvalue, and $\mathbf{e}_{i}$ is the $i$ th eigenvector. The eigen decomposition of the autoassociative matrix $\mathbf{A}$ is indicative of the statistical structure of the stimulus set used to construct it, and thus it will depend on the kinds of faces stored in the memory. Viewed in terms of eigenvectors, retrieval of a face vector from this matrix can be illustrated by rewriting Equation 2 and substituting Equation 3 for $\mathbf{A}$ as follows:

$$
\hat{\mathbf{f}}_{i}=\lambda_{1}\left(\mathbf{f}_{i} \cdot \mathbf{e}_{1}\right) \mathbf{e}_{1}+\lambda_{2}\left(\mathbf{f}_{i} \cdot \mathbf{e}_{2}\right) \mathbf{e}_{2}+\ldots+\lambda_{n}\left(\mathbf{f}_{i} \cdot \mathbf{e}_{n}\right) \mathbf{e}_{n},(4)
$$

where $\left(\mathbf{f}_{i} \cdot \mathbf{e}_{j}\right)$ is a "weight" equal to the dot product between the $i$ th face and the $j$ th eigenvector, and where $n$ is the rank of the matrix. The rank of $\mathbf{A}$ is, at most, the number of faces learned. In other words, each retrieved face can be represented by a weighted sum of eigenvectors.

The storage capacity of an autoassociative matrix is approximately $15 \%$ of its dimensionality when random (i.e., noncorrelated) vectors are used (Hopfield, 1984). ${ }^{1}$ With the face stimuli used here, two additional factors need to be taken into account. First, faces are highly correlated, so $15 \%$ will overestimate the capacity of the matrix. On the other hand, the storage capacity of this matrix was improved in the present study by applying error correction in the form of the Widrow-Hoff (i.e., delta) rule (Duda \& Hart, 1973) for learning the faces. In a neural network framework, the delta rule error correction can be implemented iteratively as follows:

$$
\mathbf{A}_{t+1}=\mathbf{A}_{t}+\gamma\left(\mathbf{f}_{i}-\mathbf{A}_{t} \mathbf{f}_{i}\right) \mathbf{f}_{i}^{T},
$$

where $\gamma$ is a learning constant that is equal to 1 divided by the iteration number. Simply seen, the matrix $\mathbf{A}$ is updated at time $t+1$ by calculating the "error," or the difference between the actual face and the model estimate at time $t,\left(\mathbf{f}_{i}-\mathbf{A}_{t} \mathbf{f}_{i}\right)$, and re-teaching this "difference" vector to the model via the outerproduct rule. This process is repeated for all faces over many iterations. As the learning progresses, the learning constant $\gamma$ decreases exponentially, and finer and finer changes are made to the matrix. Elsewhere, we have considered the error correction process, applied to images, as a kind of perceptual learning (O'Toole, Deffenbacher, et al., 1991).

From a principal components analysis point of view, the effect of this training is to equalize the eigenvalues, which is equivalent to dropping the eigenvalues from Equation 4. Viewed as an iterative process, the error correction uses the difference between the model estimate and the actual face to amplify the information that is most useful for discriminating among individual faces. This information is concentrated in the eigenvectors with smaller eigenvalues (O'Toole, Abdi, Deffenbacher, \& Valentin, in press), which explain a relatively smaller proportion of the variance for the set of faces. Hence, the error correction procedure serves to increase the importance of these eigenvectors relative to the others in retrieving a face.

Since the stimuli for this matrix are simply pixel vectors of faces, the eigenvectors of this matrix can also be interpreted as pixel vectors and can be displayed as images. Nearly all of the eigenvectors of the matrix are facelike (O'Toole \& Abdi, 1989; Sirovich \& Kirby, 1987). A face "filtered" through this matrix (via retrieval with Equation 4) is therefore expressible as a weighted sum of eigenvectors. The matrix can be thought of as a filter, because the quality of recall will be based on the statistical structure of the particular stimulus set learned. The set of weights required to reconstruct a face is referred to here as its coefficient profile. As noted by Sirovich and Kirby, the autoassociative matrix, and hence the model estimations of faces can be approximated by fewer eigenvectors, those with the larger eigenvalues. The psychological relevance of the information contained in the 
coefficient profile of a face can be seen in the usefulness of this information for categorization. For example, when the stimulus set of trained faces is a heterogeneous set of faces, such as male and female faces, or more than one race of faces, the information for determining category membership can sometimes be found in a single or a small subset of the eigenvectors. For example, O'Toole, Abdi, et al. (1991) created an autoassociative matrix by using Japanese and Caucasian faces. In a simple thresholding procedure, the second coefficient for a face (i.e., the dot product of the face and the second eigenvector) predicted race membership with $88 \%$ accuracy. An analogous situation will be demonstrated for the sex of the face.

\section{Implementation of Autoassociative Memory of Faces}

The eigenvectors and coefficient profiles of a simulation with 319 male and female Caucasian faces are provided with this program. One hundred of these faces were stored in the autoassociative matrix (i.e., were learned), whereas the remainder were "test" faces. The latter faces provide a good comparison case between the "detailed" identity-specific properties preserved for the learned faces and the general "face-like" properties that the model can reconstruct for all retrieved faces, regardless of whether or not they were learned. All faces were digitized from slides to a resolution of 16 gray levels by using a Fotovix digitizer attached to a 286-based computer with a 16-bit TARGA board (True Vision). The faces were those of young adults; roughly half were male, the other half, female. None of the slides pictured people with facial hair or glasses. The images were aligned so that all the eyes were at about the same height. The images were also cropped around the face to eliminate clothing. Each face was 151 pixels wide and 225 pixels long and thus was represented by a 33,975-pixel vector consisting of the concatenation of the pixel rows. The simulations were carried out on a Sun Microsystems SPARCstation 1 and on a Convex C-1 Vector computer.
The simulation proceeded as follows. First, Equation 1 was used to construct an autoassociative matrix for the stimulus set. The eigenvectors of the matrix were then extracted and stored in separate files. The 319 faces, some learned and some novel, were reconstructed with the Widrow-Hoff version of Equation 4. Coefficient profiles were obtained for each face and were stored in a file. An average of these coefficient profiles was also stored in a separate file.

\section{Program Description}

The associated software is intended to provide a tool for easy manipulation of the kind of information that the model makes use of in reconstructing the faces. With this tool, eigenvectors and coefficient profiles are provided for the implementation described above. ${ }^{2}$ The program has a two-stage user interface. At the highest level, the user is given a panel of buttons and text input displayed next to a multiple window display (see Figure 1). This interface can be used to:

1. initialize the graphic aspects of the program;

2. display a face, an eigenvector, or the average face;

3. specify the display of a particular face and indicate which face to display;

4. specify the display of a particular eigenvector and indicate which eigenvector to display;

5. specify the creation of a face using a subset of eigenvectors; and

6. specify an alternative set of eigenvectors to use, which one does by typing the appropriate file names into the slots provided.

At the second level of the program (see Figure 2), a panel of slider gauges indicates the preset values of the weights on the eigenvectors. These weights correspond to the choice made in the previous panel. For example, if an individual face is to be displayed, the weights are set to the values that the simulation has determined for

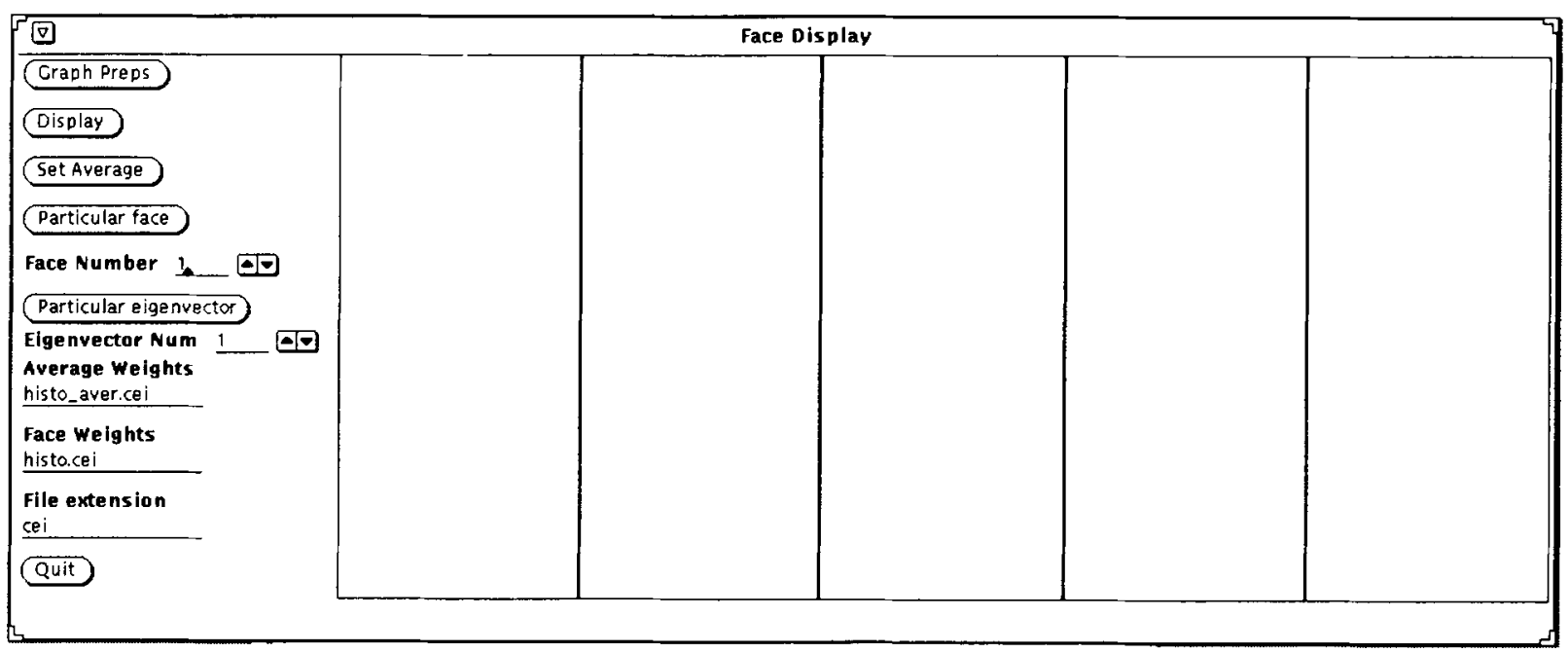

Figure 1. The top level of the program is displayed. The panel for entering control information about displaying a face, an eigenvector, or an average face appears on the left. Five empty canvases appear on the right. 


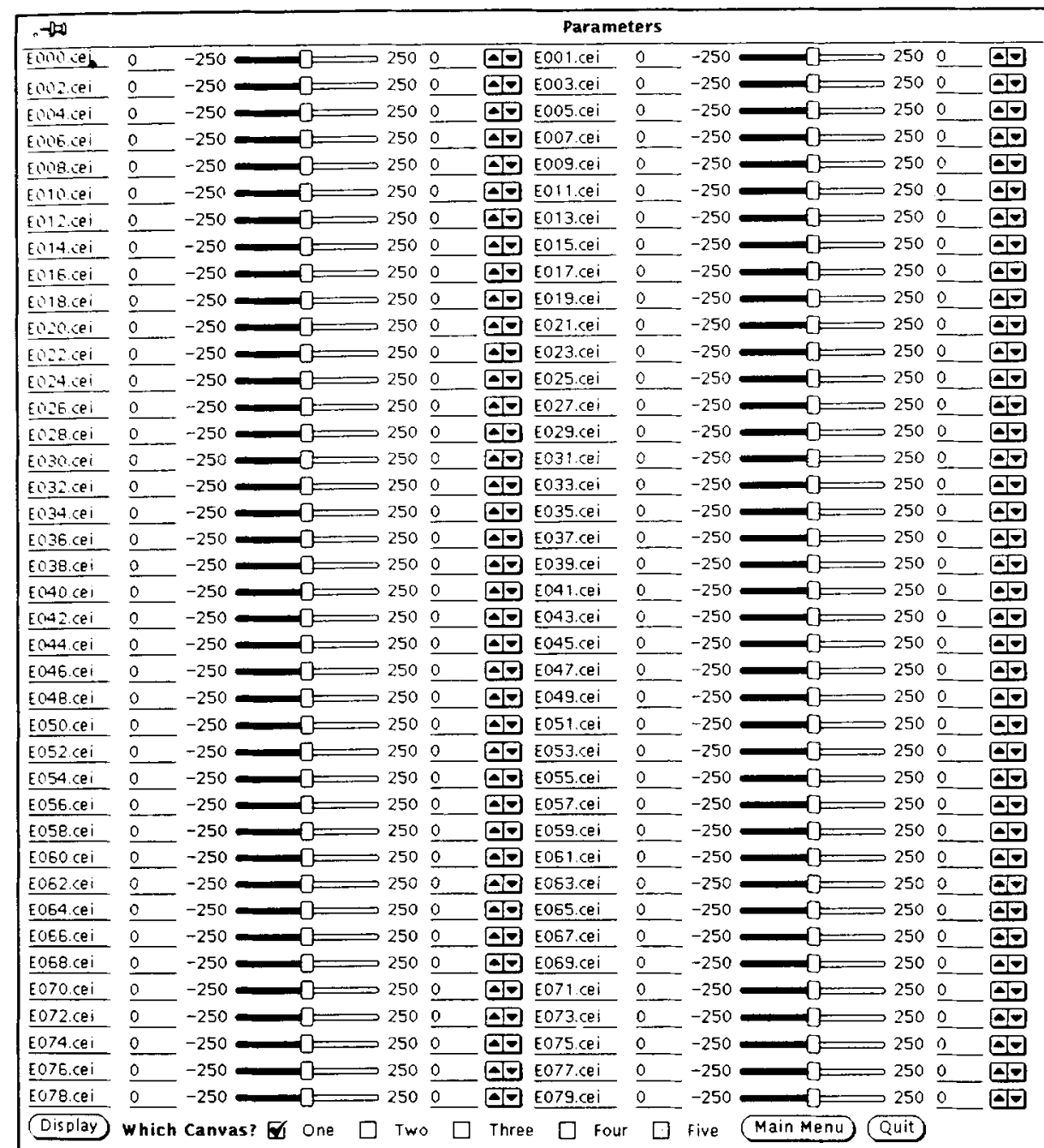

Figure 2. The parameter level of the program, with slider gauges for the first 80 eigenvectors. At the bottom of the panel, there are buttons for displaying the face according to the settings, returning to the main menu, or quitting the program. A series of check boxes are displayed for choosing the canvas on which to display the face.

that face. On the other hand, if a particular eigenvector is chosen in the previous panel, the weight on that eigenvector is set to 100 while the weights on the other eigenvectors are set to 0 . At the bottom of this panel, the user is given the choice of displaying the face or eigenvector on any one of five canvases. Thus, several faces and/or eigenvectors may be displayed simultaneously. Other buttons allow the user to: return to the main menu, quit the program, or display the "face" that is specified by the current settings of the eigenvectors. Before this display button is pressed, the user may adjust any of the eigenvector weights manually (i.e., by changing the slider values with the mouse) in order to change the appearance of the face in interesting ways. Some of these will be demonstrated later in this section. After the display button has been pressed, the program returns to its multiplewindowed output screen and draws the face on the se- lected canvas. Figure 3 illustrates the appearance of the tool when five different faces have been displayed on the five different canvases. These reconstructions used all of the eigenvectors available. Although the eigenvalues are not used in the reconstruction of the faces, the eigenvectors with the largest eigenvalues account for the largest proportion of variance in the principal components analysis of the face matrix, and hence they are usually weighted strongly in the dot product terms of Equation 4. Figure 4 displays the eigenvectors with the five largest eigenvalues, arranged according to the size of the eigenvalues. These are five of the 100 " features" used by the autoassociative memory to construct the faces displayed in Figure 3.

A final demonstration of the relevance of the program to the problem of the categorization of faces appears in Figure 5. We have observed in other simulation studies 

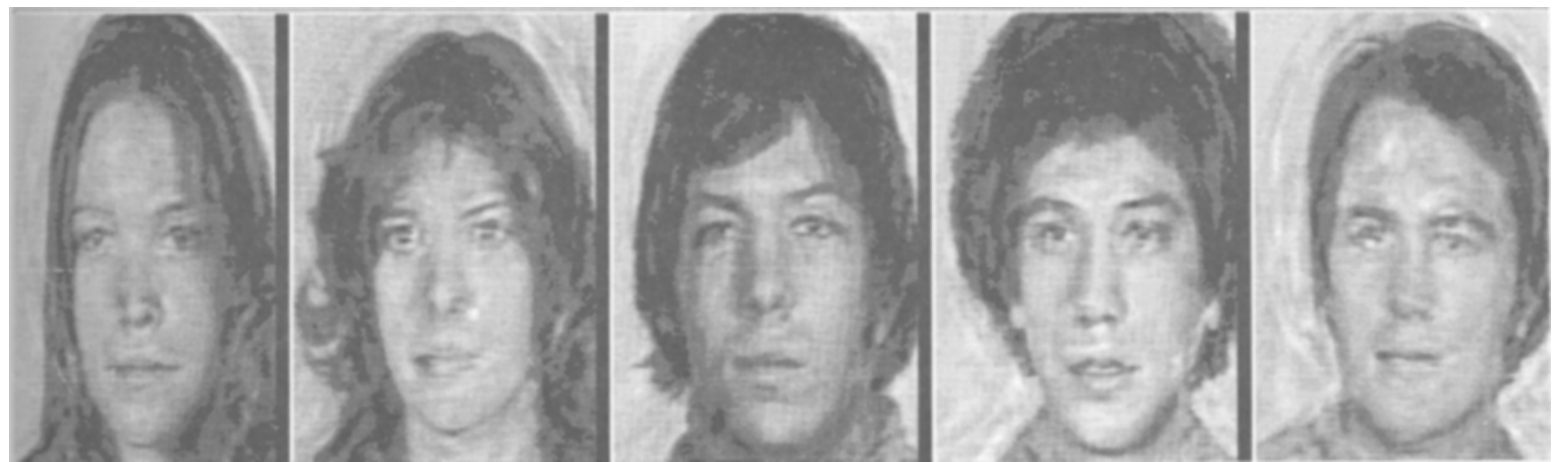

Figure 3. The top-level program canvases are pictured, with five faces. These faces were reconstructed by the simulation as a weighted sum of eigenvectors.
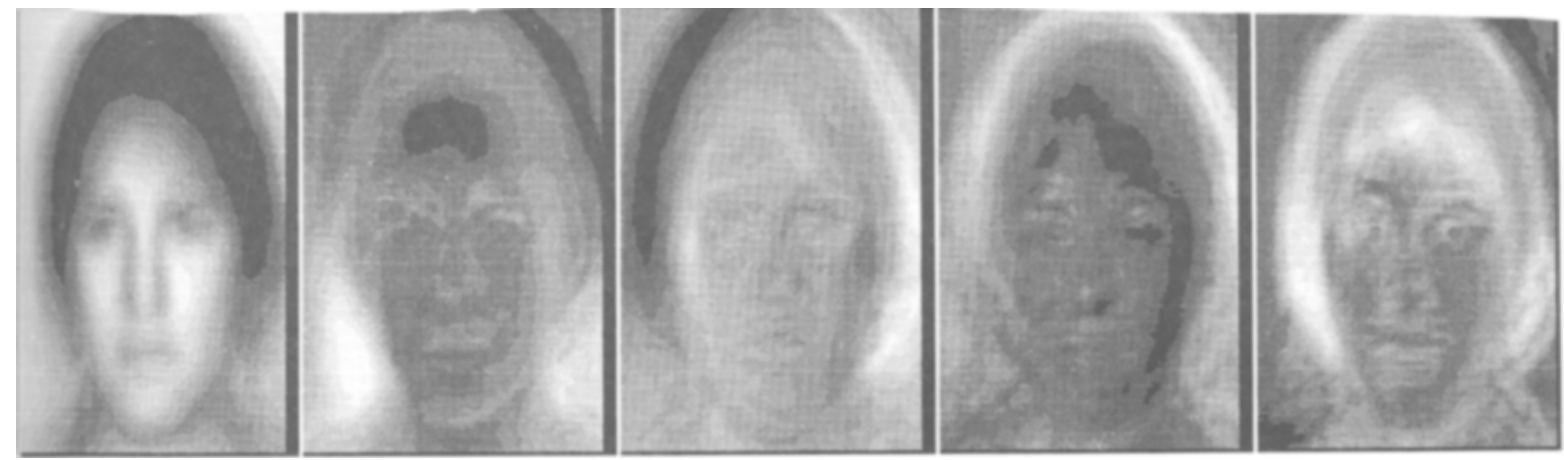

Figure 4. The top-level program canvases are pictured, with the first five eigenvectors. These are the "features" used to construct the faces displayed in Figure 4.

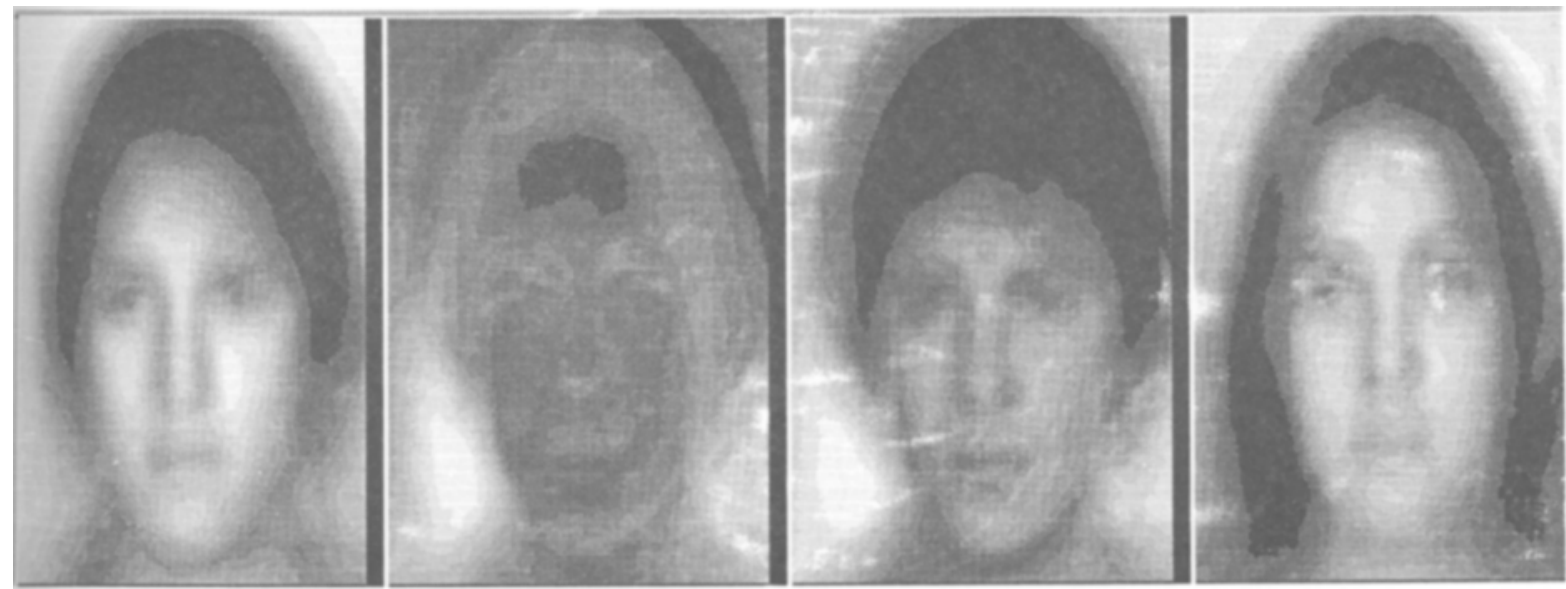

Figure 5. Canvases 1 and 2 contain the first and second eigenvectors, respectively. Canvases 3 and 4 contain the first eigenvector plus a positive and negative value of the second eigenvector, respectively. The combination face in Canvas 3 appears masculine, whereas the combination face in Canvas 4 appears feminine. 
(O'Toole, Abdi, et al., 1991; O'Toole et al., in press) that the second eigenvector is important for determining some of the gender appearance of faces, whereas the first eigenvector looks like a general "face prototype." The first two eigenvectors are displayed on the first two canvases. The result of combining these two eigenvectors by using a positive value of the second eigenvector is displayed on the third canvas. The appearance of this face is clearly masculine, and it remains masculine over a large range of positive values for the second eigenvector. The fourth canvas, by contrast, contains the first eigenvector combined with a negative value of the second eigenvector. The appearance of the resulting face is clearly feminine, and it remains feminine over a large range of negative values for the second eigenvector. Although the second eigenvector, by its appearance, does not seem to indicate information relevant to the sex of the face, in combination with the first eigenvector it controls at least part of the gender appearance of the face. It is worth noting that this is analogous to representing at least some part of the physical basis of gender in faces as a dimension in a metric multidimensional space derived from a particular set of face stimuli.

This tool has been applied recently (O'Toole et al., in press) to a systematic exploration contrasting the kinds of information present in different ranges of eigenvectors. We have found that the ranges of eigenvectors with smaller eigenvalues are optimal for recognizing faces (i.e., discriminating "learned" from "unlearned" faces), whereas the eigenvectors with larger eigenvalues are more useful for visual categorizations (e.g., sex). We also plan to apply the tool to stimulus creation for psychological experiments. Faces can be modified in various ways and saved with the standard image/screen manipulation routines available on our system (and on most others). These image files can then be used as stimuli in experimental programs to display "synthesized" faces to human observers under controlled conditions.

\section{Program Specifics}

This program is written in $\mathrm{C}$, with the $\mathrm{X}$ Window System (X11, Release 4) utilized as the window display mechanism. The $X$ Window System is an excellent development and delivery vehicle for this application, since it is vendor independent and freely available. The Massachusetts Institute of Technology's Project Athena developed X with the support of most major UNIX workstation vendors, so that it is widely available in a ready-to-run configuration on most hardware platforms. The $\mathrm{X}$ Window System is also available for IBM-PC-compatible and Apple Macintosh platforms.

The X Window System source code is obtainable free of charge via Internet (ask your System Administrator about retrieving the $\mathrm{X}$ Window System via anonymous ftp (file transfer protocol) from athena-dist.mit.edu, or any number of other sites on the Internet network). ${ }^{3}$ For platform-specific questions about the X Window System, contact your hardware vendor.

The X Window System is generally used in conjunction with platform-specific tool kits provided by hardware system vendors that make use of hardware-specific features. These tool kits and extensions supplement the lowlevel code of the $\mathrm{X}$ library routines, with higher level, easier-to-use programming libraries. Because of the diversity of platforms currently in use, we offer two versions of the program, which we hope will fit most needs. The first is written with the XView tool kit, (i.e., Sun's tool kit), to run under OpenWindows (Version 3), Sun's $\mathrm{X}$ Window System implementation. This program was used to illustrate the examples in this paper. It works only with a color/gray-scale monitor, but we also have available (upon request) another program that works on a monochrome monitor by dithering the images. The latter program has only two display windows, because of the increase in area needed to represent a dithered image. ${ }^{4}$

As previously noted, several workstation, PC, and third-party software vendors also support the use of the $X$ Window System on various platforms, including IBM, Hewlett-Packard, DEC, and Apple. Because the display mechanism of the program is handled transparently by the X Window System, the XView program execution can be displayed on any type of workstation that utilizes the $\mathrm{X}$ Window System as the display manager-that is, under any well-behaved, ICCCM compliant X Window manager, such as olwm (openlook window manager) or mwm (motif window manager). Thus, although the XViewbased program can only execute from a Sun workstation, it can be displayed on any type of workstation running $\mathrm{X}$ Windows as the display manager. This is useful if Sun workstations are used at a workplace as servers, but are placed in areas restricted to computer center personnel, or if one has any machine that runs an X Window manager, along with the possibility of accessing a Sun workstation on campus. ${ }^{5}$

Even with the transparency of the display mechanism, the different hardware platforms still limit the complete portability of the XView program. For this reason, we offer a second program that is not dependent on the XView system, but rather is written primarily with the $\mathrm{Xt}$ tool kit to give the look and feel of the interface provided by a relatively minimal number of functions in the OPENLOOK Intrinsics Toolkit (OLIT). These generic functions are available in all vendor-specific tool kits (e.g., motif tool kits). While this program is still limited in its portability because of the use of the OLIT tool kit, the generic OLIT tool kit routines can be replaced easily with the routines of tool kits available with whatever platform is in use locally.

\section{Program Availability}

The program and subsequent updates will be made available via anonymous ftp from the author at utdallas.edu, 
or by floppy disk, magnetic tape, or cassette tape. Requests for the program and data sets may be sent via electronic mail to otoole@utdallas.edu ${ }^{6}$ or by regular mail to the first author. A short user's guide is also provided with the software.

\section{REFERENCES}

Bahrick, H. P., Bahrick, P. O., \& Wittuinger, R. P. (1975). Fifty years of memory for names and faces: A cross-sectional approach. Journal of Experimental Psychology: General, 104, 54-75.

Davies, G. (1982). Composite systems for recalling faces: "Helping police with their inquiries?' In A. Trankell (Ed.), Reconstructing the past: The role of psychologists in criminal trials (pp. 299-313). Stockholm: Norstedts.

Duda, R. O., \& HaRT, P. E. (1973). Pattern classification. New York: Wiley.

Fleming, M., \& CotTrell, G. W. (1990). Categorization of faces using unsupervised feature extraction. In Proceedings of the International Joint Conference on Neural Networks, 2, 65-70.

Golomb, B. A., LAwrence, D. T., \& Sejnowski, T. J. (1991). SEXnet: A neural network identifies sex from human faces. In D. S. Touretsky \& R. Lippmann (Eds.), Advances in neural information processing systems (Vol.3, pp. 572-577). San Mateo, CA: Morgan Kaufmann.

HoPFIE LD, J. J. (1984). Neurons with graded responses have collective computational properties like those of two-state neurons. Proceedings of the National Academy of Sciences, 81, 3088-3092.

JACKSON, J. E. (1991). A user's guide to principal components analysis. New York: Wiley.

KOHONEN, T. (1977). Associative memory: A system theoretic approach. Berlin: Springer-Verlag.

OToOLE, A. J., \& ABDI, H. (1989). Connectionist approaches to visually based feature extraction. In G. Tiberghien (Ed.), Advances in cognitive psychology (Vol. 2, pp. 1-13). London: Wiley.

OToole, A. J., Abdi, H., Deffenbacher, K. A., Bartlett, J. (1991). Classifying faces by race and sex using an autoassociative memory trained for recognition. In Proceedings of the Thirteenth Annual Conference of the Cognitive Science Society (pp. 847-851). Hillsdale, NJ: Erlbaum.

O'Toole, A. J., AbDi, H., Deffenbacher, K. A., \& Valentin, D. (in press). A low dimensional representation of faces in the higher dimensions of the space. Journal of the Optical Society of America A.

O'ToOle, A. J., Deffendacher, K. A., AbDi, H., Bartlett, J. A. (1991). Simulating the "other-race effect" as a problem in perceptual learning. Connection Science Journal of Neural Computing. Artificial Intelligence, \& Cognitive Research, 3, 163-178.

O'Toole, A. J., Millward, R. B., \& Anderson, J. A. (1988). A physical system approach to recognition memory for spatially transformed faces. Neural Networks, 1, 179-199.

Sirovich, L., KiraY, M. (1987). Low-dimensional procedure for the characterization of human faces. Journal of the Optical Sociery of America A, 3, 519-524.

\section{NOTES}

1. With this "distributed" method of storing faces, the storage requirements do not increase with the number of faces learned.

2. Eigenvectors and coefficient profiles are available from a second simulation with both Caucasian and Japanese faces, upon request.

3. If a site does not have Internet access, several companies provide the X Window System on $1 / 4$-in. tapes, CD-ROM, or floppy disks for free. The MIT Software Center has details on these services. Contact: MIT Software Center, W32-300, 20 Carlton Street, Cambridge, MA 02139; (617) 253-7686.

4. Dithering or half-toning works by using a small number of pixels, for example a $4 \times 4$ grid, to display gray levels. The maximum luminance value would be achieved by lighting all 16 pixels, whereas half this value would be achieved by randomly lighting 8 of the 16 pixels, and so forth.

5. Technically speaking, the program can be run from anywhere via ethernet. In fact, the present program was demonstrated recently during a talk given in California, by using the $\mathrm{X}$ Window display manager on the DEC Workstation there, run from a SPARCstation in Texas. This procedure, however, is not advisable, because it is bandwidth intensive for the network.

6. One can send binaries, such as the faces or executable programs, via e-mail if one encodes and compresses them. This, however, is generally not advisable, since it may pose data-overflow problems for some mailers.

(Manuscript received October 1, 1991; revision accepted for publication October 23, 1992.) 